TITLE:

\title{
Comparison of Rotational Hysteretic Properties of Isotropic Vector Stop Models
}

$\operatorname{AUTHOR}(S)$ :

Matsuo, Tetsuji

CITATION:

Matsuo, Tetsuji. Comparison of Rotational Hysteretic Properties of Isotropic Vector Stop Models. IEEE TRANSACTIONS ON MAGNETICS 2009, 45(3): 1194-1197

\section{ISSUE DATE:}

2009-03

URL:

http://hdl.handle.net/2433/109811

\section{RIGHT:}

(c) 2009 IEEE. Personal use of this material is permitted. However, permission to reprint/republish this material for advertising or promotional purposes or for creating new collective works for resale or redistribution to servers or lists, or to reuse any copyrighted component of this work in other works must be obtained from the IEEE. 


\title{
Comparison of Rotational Hysteretic Properties of Isotropic Vector Stop Models
}

\author{
Tetsuji Matsuo \\ Department of Electrical Engineering, Kyoto University, Kyoto 615-8510, Japan
}

\begin{abstract}
Two isotropic vector stop models are compared. One is a superposition of scalar stop models and the other uses a geometrically vectorized stop hysteron. Their simulated rotational hysteresis losses are adjusted to a measured loss. The latter adjusted vector stop model accurately represents the rotational hysteretic property.
\end{abstract}

Index Terms-Isotropic vector hysteresis, rotational hysteresis loss, silicon steel sheet, superposition, vector stop hysteron.

\section{INTRODUCTION}

$\mathbf{T}$ HE stop model [1]-[3] is an efficient and precise hysteresis model that directly provides a hysteretic output of magnetic field from an input of magnetic flux density. The model has two vector versions: one version is the superposition of scalar models along the azimuthal direction [4], [5], whereas the other uses a geometrically vectorized stop hysteron [1], [6], [7]. The latter is more efficient than the former, which requires azimuthal integration.

Reference [7] discussed the properties of the latter vector model and a similar vector model using a vectorized play hysteron comparing the measured rotational hysteretic properties of a silicon steel sheet. The comparison showed that the geometrically vectorized stop model yields larger rotational hysteresis loss than does the measured one. Accordingly, the vector stop model requires a loss adjustment, which has been applied to vector play models successfully [8].

On the other hand, reference [5] generalized the vector stop model of superposition type and showed its potential to control the rotational property. However, it has not yet been verified by comparing with the measured vector hysteretic property.

This paper compares rotational hysteretic properties of the two vector stop models with measured properties of silicon steel sheets. A direct method is proposed, which can adjust the simulated rotational hysteresis loss to a measured loss without affecting the unidirectional representation of the vector models.

\section{SCALAR AND Vector Stop Models}

\section{A. Scalar Stop Model}

A scalar stop model [3] describes a hysteretic relation between an input of magnetic flux density $B$ and an output of magnetic field $H$ as

$$
H=S(B)=\int_{0}^{B_{\mathrm{S}}} g\left(\eta, s_{\eta}(B)\right) \mathrm{d} \eta
$$

Manuscript received October 07, 2008. Current version published February 19, 2009. Corresponding author: T. Matsuo (e-mail: tmastuo@kuee.kyoto-u.ac. jp).

Digital Object Identifier 10.1109/TMAG.2009.2012555 where $s_{\eta}$ is a stop hysteron operator having height $\eta, g$ is an input-independent shape function, and $B_{\mathrm{S}}$ is the saturation magnetic flux density. The stop hysteron operator $s_{\eta}$ is given as

$$
s_{\eta}(B)=\max \left(\min \left(B-B^{0}+s_{\eta}^{0}, \eta\right),-\eta\right)
$$

or

$$
s_{\eta}(B)=\max \left(\min \left(B-p_{\eta}^{0 *}, \eta\right),-\eta\right) .
$$

Therein, $B^{0}$ and $s_{\eta}^{0}$ are the values of $B$ and $s_{\eta}$ at the previous time point; $p_{\eta}^{0 *}$ is defined as

$$
p_{\eta}^{0 *}=\max \left(\min \left(B^{0}-s_{\eta}^{0}, B_{\mathrm{S}}-\eta\right),-B_{\mathrm{S}}+\eta\right) .
$$

The stop model (1) with hysteron (2) exhibits a hysteretic property for $|B|>B_{\mathrm{S}}$ whereas hysteron (3) prohibits the hysteretic property after saturation.

The parameter $B_{\mathrm{S}}$ should be determined from the saturation magnetization of the simulated material. However, from a practical view point, $B_{\mathrm{S}}$ is determined from the maximum flux density of available measured data.

\section{B. Superposition of Scalar Stop Models}

Similarly to the vector Preisach model proposed by Mayergoyz [9], a 2-D isotropic vector stop model can be constructed using the superposition of scalar stop models [4], [5]

$$
\boldsymbol{H}=\boldsymbol{S}_{\mathrm{S}}(\boldsymbol{B})=\int_{-\pi / 2}^{\pi / 2} \boldsymbol{e}_{\varphi} S_{2}\left(|\boldsymbol{B}| \cos ^{\frac{1}{n}}(\theta-\varphi)\right) \mathrm{d} \varphi .
$$

Here, $S_{2}(B)$ is a scalar stop model using (3), $\boldsymbol{e}_{\varphi}$ is the unit vector along the $\varphi$-direction, $\theta$ is the angle of $\boldsymbol{B}$, and $n$ is a projection parameter for input $\boldsymbol{B}$ to the $\varphi$-direction; $\cos ^{1 / n} \phi$ denotes $|\cos \phi|^{1 / n} \operatorname{sign}(\cos \phi)$ for simplicity of expression. Reference [5] gives the identification method of the vector model (5) and discusses the rotational hysteretic property of (5).

\section{Vectorization of the Stop Hysteron}

The stop hysteron (2) is geometrically vectorized [6], [7] as

$$
\boldsymbol{s}_{\eta}(\boldsymbol{B})=\frac{\eta\left(\boldsymbol{B}-\boldsymbol{p}_{\eta}^{0}\right)}{\max \left(\eta,\left|\boldsymbol{B}-\boldsymbol{p}_{\eta}^{0}\right|\right)}
$$

where $\boldsymbol{p}_{\eta}^{0}$ is the vector $\boldsymbol{p}_{\eta}=\boldsymbol{B}-\boldsymbol{s}_{\eta}$ at the previous time point. (See the Appendix for the geometrical meaning of the vector stop hysteron.) 

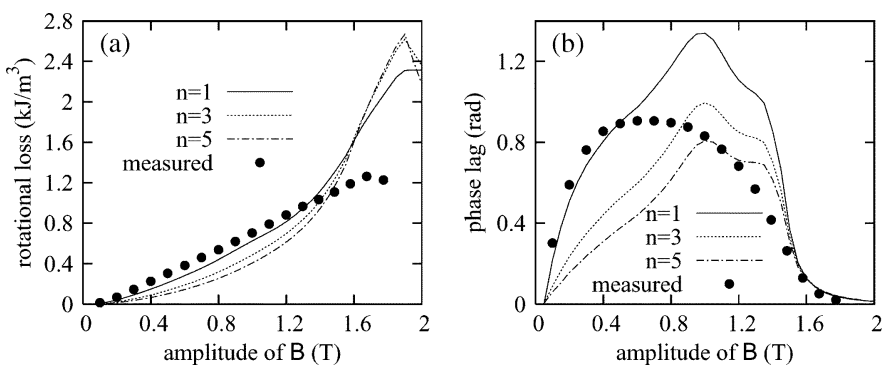

Fig. 1. Rotational properties of the vector stop model $\boldsymbol{S}_{\mathrm{S}}$ : (a) rotational hysteresis loss and (b) phase lag of $\boldsymbol{B}$ to $\boldsymbol{H}$.

Using this vector stop hysteron, a vector stop model [7] is given as

$$
\begin{aligned}
\boldsymbol{H} & =\boldsymbol{S}_{\mathrm{G}}(\boldsymbol{B})=\int_{0}^{B_{\mathrm{S}}} \boldsymbol{g}\left(\eta, \boldsymbol{s}_{\eta}(\boldsymbol{B})\right) \mathrm{d} \eta \\
\boldsymbol{g}(\eta, \boldsymbol{s}) & =g(\eta,|\boldsymbol{s}|) \frac{\boldsymbol{s}}{|\boldsymbol{s}|} .
\end{aligned}
$$

An anisotropic version of the vector stop model has been discussed in [6]. This vector model is simpler than the vector model (5) having an integration along the $\varphi$-direction, which is computationally expensive.

The vector model (7) using (6) exhibits a hysteretic property even when $|B|>B_{\mathrm{S}}$. To prohibit the hysteretic property after the saturation, $\boldsymbol{s}_{\eta(\boldsymbol{B})}$ is redefined similarly to (3) as

$$
\boldsymbol{s}_{\eta}(\boldsymbol{B})=\frac{\eta\left(\boldsymbol{B}-\boldsymbol{p}_{\eta}^{0 *}\right)}{\max \left(\eta,\left|B-\boldsymbol{p}_{\eta}^{0 *}\right|\right)}
$$

where

$$
\boldsymbol{p}_{\eta}^{0 *}=\frac{\left(B_{\mathrm{S}}-\eta\right)\left(\boldsymbol{B}^{0}-\boldsymbol{s}_{\eta}^{0}\right)}{\max \left(B_{\mathrm{S}}-\eta,\left|\boldsymbol{B}^{0}-\boldsymbol{s}_{\eta}^{0}\right|\right)} .
$$

The stop hysteron (9) is equivalent to (3) for unidirectional inputs. The behaviors of vector stop hysterons (6) and (9) are compared in [7].

For unidirectional inputs, the vector model (7) using (6) or (9) is reduced to the scalar model (1) with (2) or (3), respectively, having the same shape function $g(\eta, s)$. This means that $g(\eta, s)$ can be determined only from unidirectional measured data.

The rotational hysteretic property of the vector stop model using (6) or (9) for rotational input is discussed in [7].

\section{COMPARISON OF VECTOR MODELS}

\section{A. Identification From Unidirectional Property}

The hysteretic properties of a nonoriented silicon steel sheet, JIS 50A1300, are measured using a rotational single sheet tester. The anisotropic property is averaged along the azimuthal direction to obtain an isotropic property for numerical simulations.

First, the scalar stop model (1) discretized with 38 hysterons is identified using the method described in [4] using unidirectional (alternating) BH loops. Owing to the lack of measured
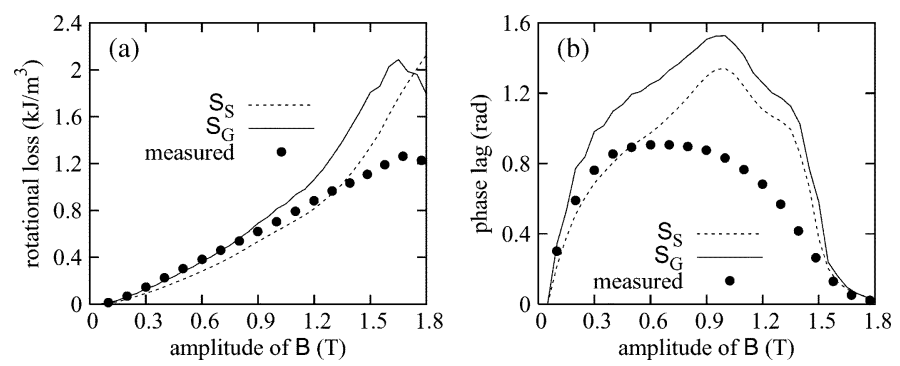

Fig. 2. Comparison of rotational properties of $\boldsymbol{S}_{\mathrm{G}}$ with $\boldsymbol{S}_{\mathrm{S}}$ : (a) rotational hysteresis loss and (b) phase lag of $\boldsymbol{B}$ to $\boldsymbol{H}$.

data greater than $1.9 \mathrm{~T}, B_{\mathrm{S}}$ is set to $1.9 \mathrm{~T}$ despite this being small compared with the saturation magnetization of the silicon steel sheet. The shape function of the scalar model is used directly for $\boldsymbol{S}_{\mathrm{G}}$. The vector model $\boldsymbol{S}_{\mathrm{S}}$ is identified from the scalar model [5]. The integration along the $\varphi$-direction is performed using the midpoint rule with 40 integration points. Because of the azimuthal integration, $\boldsymbol{S}_{\mathrm{S}}$ requires about 14 times the computation time of $\boldsymbol{S}_{\mathrm{G}}$.

\section{B. Vector Property for Rotational Input}

Vector hysteretic properties of the vector models for rotational input

$$
\boldsymbol{B}=\left(B_{\mathrm{a}} \cos \omega t, B_{\mathrm{a}} \sin \omega t\right)
$$

are examined. Here $B_{\mathrm{a}}$ is the amplitude of $\boldsymbol{B}$.

Fig. 1 shows the (rate-independent) rotational hysteresis loss and phase lag of $\boldsymbol{B}$ to $\boldsymbol{H}$ yielded by $\boldsymbol{S}_{\mathrm{S}}$ with $n=1,3,5$, where the measured rotational hysteresis loss is also shown for comparison. The measured average phase lag $\beta_{\text {lag }}$ is given as

$$
\beta_{\operatorname{lag}}=\tan ^{-1} \frac{\left|\int_{0}^{2 \pi} \boldsymbol{H} \times \boldsymbol{B} \mathrm{d}(\omega t)\right|}{\int_{0}^{2 \pi} \boldsymbol{H} \cdot \boldsymbol{B} \mathrm{d}(\omega t)} .
$$

The rotational hysteresis loss with $n=1$ saturates whereas the rotational loss $n>1$ decreases when $B_{\mathrm{a}}>B_{\mathrm{S}}=1.9 \mathrm{~T}$. However, the measured rotational loss begins to decrease at $B$ less than 1.9 T. On the other hand, $B_{\mathrm{S}}$ used in this simulation is smaller than the alternating hysteresis loss of general silicon steel sheet saturates. This means that altering only $n$ and $B_{\mathrm{S}}$ cannot sufficiently control the saturation property represented by $\boldsymbol{S}_{\mathrm{S}}$.

Fig. 2 compares the rotational hysteresis losses and the phase lag of $\boldsymbol{B}$ to $\boldsymbol{H}$ given by $\boldsymbol{S}_{\mathrm{G}}$ and $\boldsymbol{S}_{\mathrm{S}}(n=1)$. The rotational loss given by $\boldsymbol{S}_{\mathrm{G}}$ begins to decrease before the amplitude $B_{\mathrm{a}}$ becomes $B_{\mathrm{S}}$. However, $\boldsymbol{S}_{\mathrm{G}}$ gives rotational loss and phase lag that are larger than the measured values.

\section{Adjustment of Rotational Loss Using a Weighting Function}

The vector stop models having weighting functions are given as

$$
\boldsymbol{S}_{\mathrm{Sw}}(\boldsymbol{B})=w(|\boldsymbol{B}|) \boldsymbol{S}_{\mathrm{S}}(\boldsymbol{B}), \quad \boldsymbol{S}_{\mathrm{Gw}}(\boldsymbol{B})=w(|\boldsymbol{B}|) \boldsymbol{S}_{\mathrm{G}}(\boldsymbol{B})
$$

where $w(B)$ is the weighting function. When $w(B)$ is given, $\boldsymbol{S}_{\mathrm{S}}(\boldsymbol{B})$ and $\boldsymbol{S}_{\mathrm{G}}(\boldsymbol{B})$ are identified from $H(B) / w(B)$ where 

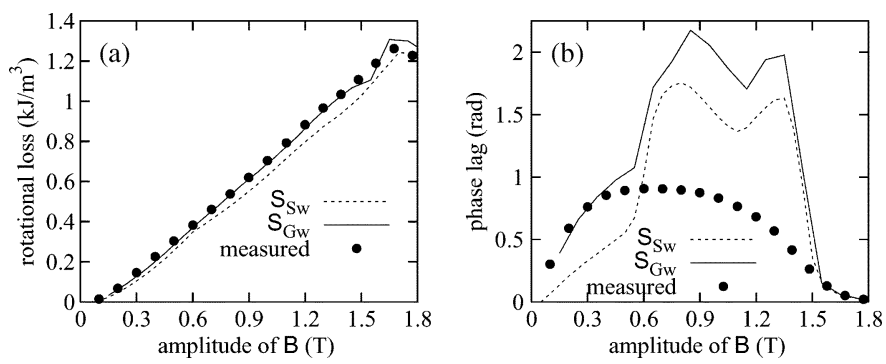

Fig. 3. Rotational properties of $\boldsymbol{S}_{\mathrm{Sw}}$ and $\boldsymbol{S}_{\mathrm{Gw}}$ adjusted by the weighting function: (a) rotational hysteresis loss and (b) phase lag of $B$ to $H$.

(a)

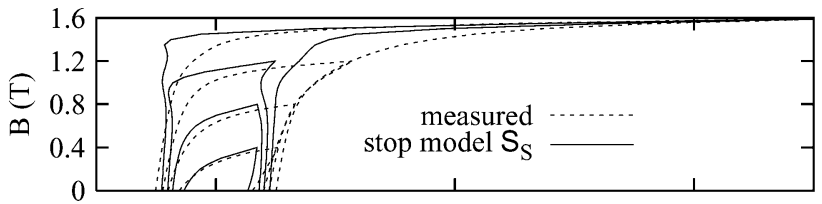

(b)

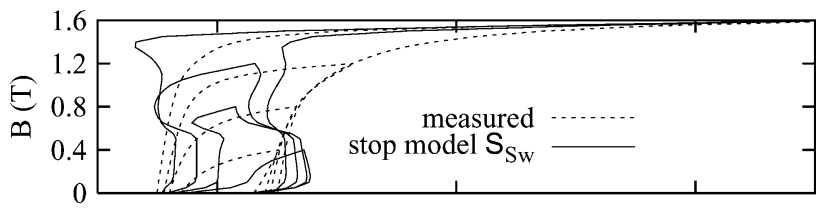

(c)

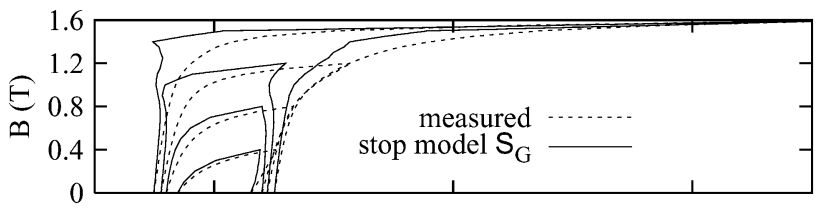

(d)

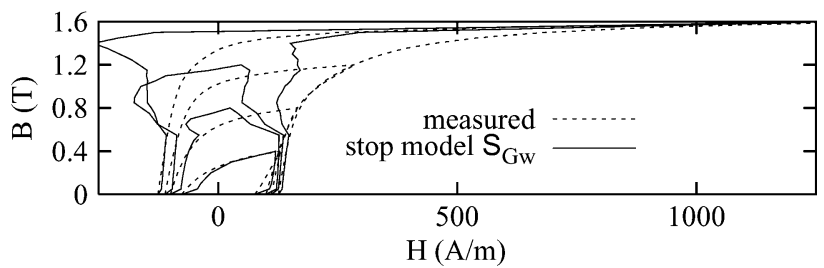

Fig. 4. Unidirectional BH loops: (a) $\boldsymbol{S}_{\mathrm{S}}(w(B)=1)$, (b) $\boldsymbol{S}_{\mathrm{Sw}}$ (loss adjusted), (c) $\boldsymbol{S}_{\mathrm{G}}(\mathrm{w}(\mathrm{B})=1)$, and (d) $\boldsymbol{S}_{\mathrm{Gw}}$ (loss adjusted).

$H(B)$ is a measured unidirectional hysteretic property. Reference [8] proposed a method for adjusting the simulated rotational loss to a measured loss using the weighting function. The weighting function is iteratively modified by

$$
w^{k+1}(B)=\frac{L_{\mathrm{rot}}^{\text {measured }}(B)}{L_{\mathrm{rot}}^{k}(B)} w^{k}(B)
$$

where $L_{\text {rot }}^{\text {measured }}(B)$ is the measured rotational loss for amplitude $B, L_{\text {rot }}^{k}(B)$ is the computed rotational loss given by the weighting function for the $k$ th iteration, and $w^{0}(B)=1$. Usually, about five iterations are sufficient for $L_{\text {rot }}^{k}(B)$ to converge. Fig. 3 shows the rotational losses and the phase lags given by $\boldsymbol{S}_{\mathrm{Sw}}$ and $\boldsymbol{S}_{\mathrm{Gw}}$ with $w^{5}(B)$, where $L_{\text {rot }}^{5}(B)$ almost coincides with the measured loss. However, the modified $w(B)$ enlarges the discrepancy between simulated and measured phase lags. Fig. 4 shows unidirectional properties of the stop models where the modified $w(B)$ deteriorates the representation accuracy of $\mathrm{BH}$ loops, which are greatly affected by the weighting function
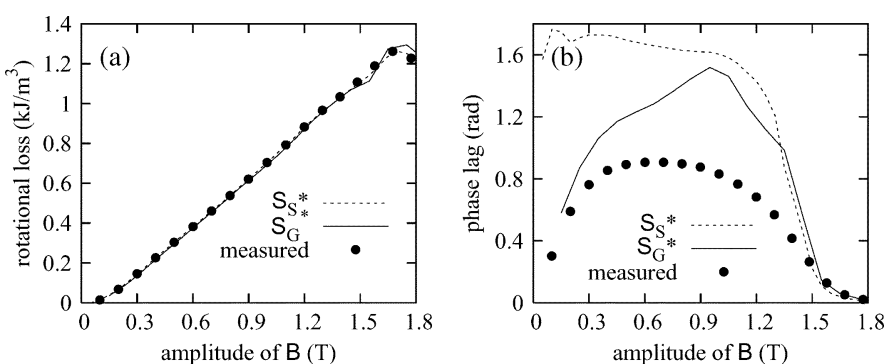

Fig. 5. Rotational properties of $\boldsymbol{S}_{\mathrm{Sw}}^{*}$ and $\boldsymbol{S}_{\mathrm{Gw}}^{*}$ adjusted by (15) with $w(B)=$ 1: (a) rotational hysteresis loss and (b) phase lag of $\boldsymbol{B}$ to $\boldsymbol{H}$.

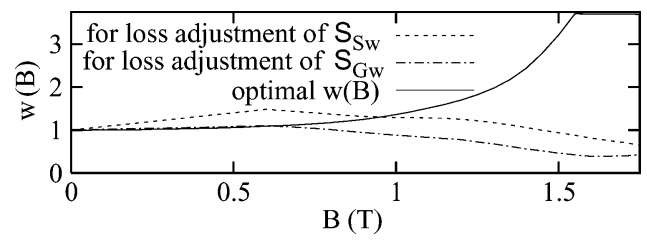

Fig. 6. Weighting functions.

[3], [10]. In contrast, unidirectional symmetric BH loops represented by the vector play model [8] are not affected by the weighting function when the play model is identified from the symmetric $\mathrm{BH}$ loops.

\section{Direct Adjustment of Rotational Loss}

This paper proposes a direct method to adjust the simulated rotational loss to a measured one that allows a free choice of the weighting function.

A newly modified stop model $\boldsymbol{S}_{\mathrm{Gw}}^{*}$ is given by

$$
\boldsymbol{S}_{\mathrm{Gw}}^{*}=\left(\boldsymbol{S}_{\mathrm{Gw}} \cdot \boldsymbol{e}_{/ /}\right) \boldsymbol{e}_{/ /}+r(|\boldsymbol{B}|)\left(\boldsymbol{S}_{\mathrm{Gw}} \cdot \boldsymbol{e}_{\perp}\right) \boldsymbol{e}_{\perp}
$$

where $\boldsymbol{e}_{/ /}$and $\boldsymbol{e}_{\perp}$ are parallel and perpendicular unit vectors to $\boldsymbol{B}$, respectively, and $r(|\boldsymbol{B}|)$ is the ratio given as the following:

$$
r(B)=\frac{L_{\mathrm{rot}}^{\text {measured }}(B)}{L_{\mathrm{rot}}^{\mathrm{Gw}}(B)}
$$

where $L_{\mathrm{rot}}^{\mathrm{Gw}}(B)$ is the simulated rotational loss given by $\boldsymbol{S}_{\mathrm{Gw}}$. The stop model $\boldsymbol{S}_{\mathrm{Sw}}$ is similarly modified. This modification does not affect the unidirectional properties of $\boldsymbol{S}_{\mathrm{Gw}}$ and $\boldsymbol{S}_{\mathrm{Sw}}$ because $\boldsymbol{e}_{\perp}$ becomes zero. Accordingly, $w(B)$ is determined to improve the unidirectional representation accuracy.

Fig. 5 shows the rotational hysteresis losses and phase lags given by $\boldsymbol{S}_{\mathrm{Sw}}^{*}$ and $\boldsymbol{S}_{\mathrm{Gw}}^{*}$ with $w(B)=1$. The modified stop models obtain accurate rotational losses.

An optimal weighting function is determined by the method proposed in [10] using unidirectional symmetric $\mathrm{BH}$ loops. Fig. 6 shows the optimal weighting function comparing with $w(B)$ for loss adjustment of $\boldsymbol{S}_{\mathrm{Sw}}$ and $\boldsymbol{S}_{\mathrm{Gw}}$. Fig. 7 shows the rotational losses and phase lags given by adjusted $\boldsymbol{S}_{\mathrm{Sw}}^{*}$ and $S_{\mathrm{Gw}}^{*}$ with the optimal weighting function. Fig. 8 presents unidirectional BH loops simulated by the stop models with the optimal weighting function. The adjusted stop models obtain accurate rotational losses and unidirectional BH loops. The phase lag representation is also improved by $\boldsymbol{S}_{\mathrm{Gw}}^{*}$ whereas $\boldsymbol{S}_{\mathrm{Sw}}^{*}$ fails to represent it accurately. 

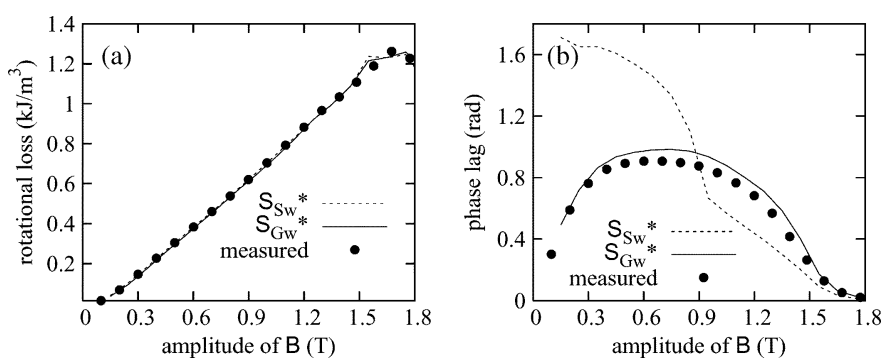

Fig. 7. Rotational properties of $\boldsymbol{S}_{\mathrm{Sw}}^{*}$ and $\boldsymbol{S}_{\mathrm{Gw}}^{*}$ adjusted by (15) with optimal $w(B)$ : (a) rotational hysteresis loss and (b) phase lag of $\boldsymbol{B}$ to $\boldsymbol{H}$.

(a)

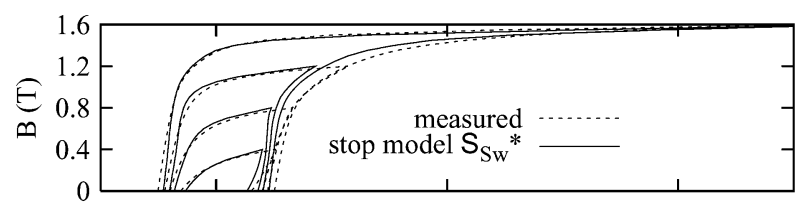

(b)

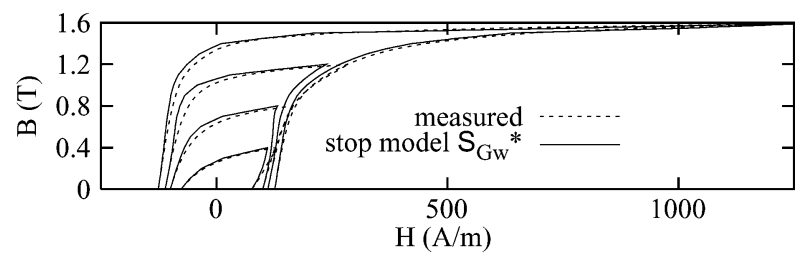

Fig. 8. Unidirectional BH loops obtained with optimal $w(B)$ : (a) $\boldsymbol{S}_{\mathrm{Sw}}^{*}$ and (b) $S_{\mathrm{Gw}}^{*}$.
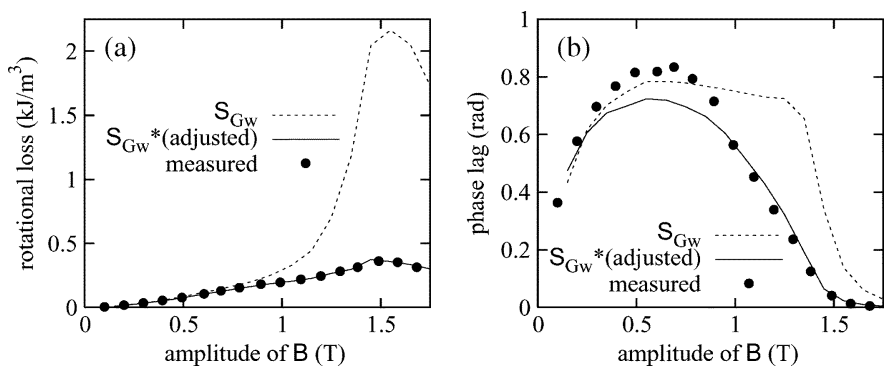

Fig. 9. Simulation for JIS $35 \mathrm{~A} 300$ by $S_{\mathrm{Gw}}$ and $S_{\mathrm{Gw}}^{*}$ with optimal $w(B)$ : (a) rotational hysteresis loss and (b) phase lag of $\boldsymbol{B}$ to $\boldsymbol{H}$.
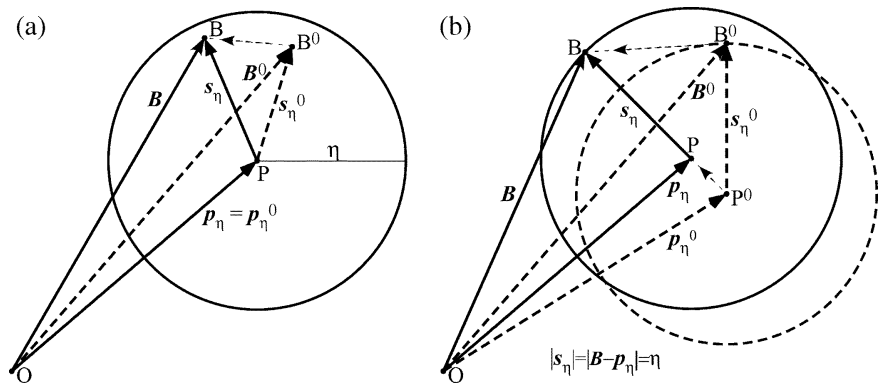

Fig. 10. Behavior of the vector stop hysteron: (a) $\left|\boldsymbol{B}-\boldsymbol{p}_{\eta}^{0}\right| \leq \eta$ and (b) $\mid \boldsymbol{B}-$ $\boldsymbol{p}_{\eta}^{0} \mid>\eta$.

\section{E. Examination of Another Steel}

The vector stop model $\boldsymbol{S}_{\mathrm{Gw}}$ is examined for another silicon steel sheet, JIS 35A300. Fig. 9 shows the rotational hysteretic property simulated by $\boldsymbol{S}_{\mathrm{Gw}}$ and the adjusted $\boldsymbol{S}_{\mathrm{Gw}}^{*}$ with the optimal weighting function. The direct method (15) effectively adjusts the rotational loss without large discrepancy of the phase lag.

\section{CONCLUSION}

Two isotropic vector stop models are compared. One is represented by a superposition of scalar stop models, whereas the other is given by a geometrical vectorization of a stop hysteron. The latter model is more efficient than the former, which requires azimuthal integration.

The rotational loss adjustment using the weighting function deteriorates the representation of the unidirectional hysteresis properties of vector stop models.

Another adjusting method is proposed, which can directly control the rotational hysteresis loss without affecting the unidirectional representations of the vector models. The proposed method allows the vector stop models to use optimal weighting functions for accurate representation of the unidirectional property. The adjusted geometrically vectorized stop model accurately represents the rotational hysteretic property.

\section{APPENDIX \\ GeOMETRICAL MEANing OF VeCtOR StOp Hysteron}

Point $\mathrm{B}(\overrightarrow{\mathrm{OB}}=\boldsymbol{B})$ in Fig. 10 is located in a circle for which the center and radius are $\mathrm{P}\left(\overrightarrow{\mathrm{OP}}=\boldsymbol{p}_{\eta}\right)$ and $\eta$, respectively. When B moves inside the circle, P does not move [Fig. 10(a)]. When $B$ reaches the circumference of the circle, the circle and its center P move together with B [Fig. 10(b)]. The stop hysteron $\boldsymbol{s}_{\eta}=B-\boldsymbol{p}_{\eta}$ is given by $\overrightarrow{\mathrm{PB}}$.

\section{ACKNOWLEDGMENT}

The author would like to thank his graduate student $\mathrm{H}$. Kaneda, Kyoto University, Japan, for measurement of the magnetic properties of silicon steel sheets. This work was supported in part by the Japan Society for the Promotion of Science, Grant-in-Aid for Scientific Research (C), 19560288.

\section{REFERENCES}

[1] M. A. Krasnosel'skii and A. V. Pokrovskii, Systems With Hysteresis. Berlin, Germany: Springer-Verlag, 1989.

[2] S. Bobbio, G. Miano, C. Serpico, and C. Visone, "Models of magnetic hysteresis based on play and stop hysterons," IEEE Trans. Magn., vol. 33, pp. 4417-4426, Nov. 1997.

[3] T. Matsuo, Y. Terada, and M. Shimasaki, "Stop model with input-dependent shape function and its identification methods," IEEE Trans. Magn., vol. 40, pp. 1776-1783, Jul. 2004.

[4] T. Matsuo and M. Shimasaki, "Isotropic vector hysteresis represented by superposition of stop hysteron models," IEEE Trans. Magn., vol. 37, pp. 3357-3360, Sep. 2001.

[5] T. Matsuo and M. Shimasaki, "Generalization of an isotropic vector hysteresis model represented by the superposition of stop models-Identification and rotational hysteresis Loss-," IEEE Trans. Magn., vol. 43, pp. 1389-1392, Apr. 2007.

[6] J. V. Leite, N. Sadowski, P. Kuo-Peng, and J. P. A. Bastos, "A new anisotropic vector hysteresis model based on stop hysterons," IEEE Trans. Magn., vol. 41, pp. 1500-1503, May 2005.

[7] T. Matsuo, "Rotational saturation properties of isotropic vector hysteresis models using vectorized stop and play hysterons," IEEE Trans. Magn., vol. 44, no. 11, pp. 3185-3188, Nov. 2008.

[8] T. Matsuo and M. Shimasaki, "Two types of isotropic vector play models and their rotational hysteresis losses," IEEE Trans. Magn., vol. 44, no. 6, pp. 898-901, Jun. 2008.

[9] I. D. Mayergoyz, Mathematical Models of Hysteresis and Their Applications. New York: Elsevier, 2003.

[10] T. Matsuo and M. Shimasaki, "A method for optimal identification of a stop model with input-dependent shape function," IEEE Trans. Magn., vol. 42, pp. 3818-3824, Dec. 2006. 